LBNL-42165

August 1998

\title{
The final-state interaction phases of the two-body $B$ decay: The bounds from the updated data
}

\author{
Mahiko Suzuki \\ Department of Physics and Lawrence Berkeley National Laboratory \\ University of California, Berkeley, California 94720
}

\begin{abstract}
The updated experimental data are used to analyze the final-state interaction phases of the two-body decay amplitudes of the $B$ mesons, $B \rightarrow \bar{D} \pi, \bar{D} \rho$, $\bar{D}^{*} \pi$, and $\bar{D}^{*} \rho$. Combining the upper bounds on the branching fractions of the color-suppressed neutral modes with those of the charged modes, we have set constraints on the relative phases between the amplitudes $A\left(B^{0} \rightarrow X^{-} Y^{+}\right)$ and $A\left(B^{+} \rightarrow X^{0} Y^{+}\right)$where $X=\bar{D}$ or $\bar{D}^{*}$ and $Y=\pi$ or $\rho$. The numbers that we have obtained point to small final-state interactions. When these relative phases are expressed in those of the isospin amplitudes, the bounds become less tight since the experimental errors accumulate. In the decay where many multibody channels are open, however, there is little advantage in breaking up the observed amplitudes into the isospin eigenchannels for analysis of the final-state interactions.
\end{abstract}

13.20.He, 13.25.-k, 14.40.Nd

Typeset using REVTEX 


\section{INTRODUCTION}

It is important to know in the nonleptonic decay of the $\mathrm{B}$ mesons how much phase is generated for the decay amplitudes by the final-state interaction. Many calculations were made on the short-distance effects assuming that the long-distance effects be small or simply ignoring them [1]. Over the years various arguments have been presented in support of small to vanishing long-distance phases for the two-body decay 2 - 4 . Since there is no method to compute the long-distance effects accurately, some warned about the possibility of large phases [5]. Experimentally, persistence of the color suppression is one strong qualitative evidence for the small phases. To learn about the final-state interaction phases of the $B$ decay, we have analyzed here the recently updated data [6] on the the two-body decay modes. Specifically we have chosen the decay modes $B \rightarrow \bar{D} \pi, \bar{D}^{*} \pi, \bar{D} \rho$, and $\bar{D}^{*} \rho$, which proceed through the nonpenguin interactions. With the current experimental uncertainties, the data are consistent with vanishing dynamical phases in all cases. Thanks to the substantial improvement in the accuracy of measurement, however, our analysis sets the meaningful upper bounds on the relative phases of the decay amplitudes. The most stringent bound has been set at the level of $10^{\circ}$.

Before starting, we would like to point out significance and insignificance of the isospin amplitudes in the nonleptonic decays. In the decays where only a small number of decay channels are open, analyzing the isospin amplitudes has a clear advantage. In the extreme case where only the state $A B$ and its isospin-related states are allowed, we should study their isospin eigenstates since the decay amplitudes of definite isospin carry the strong interaction eigenphases of elastic $A B$ scattering [8]. When another final state $C D$ exists and couples to $A B$, it still makes sense to analyze the $2 \times 2$ S-matrix of $A B$ and $C D$ with definite isospin. However, the advantage disappears when more than a few channels is open and a channel coupling occurs in the final state. In this case the strong interaction S-matrix is an $N \times N$ matrix $(N \gg 1)$. In terms of the eigenphase shifts $\delta_{a}$ defined by $\langle b|S| a\rangle=$ $\delta_{b a} e^{2 i \delta_{a}}(a, b=1,2,3, \cdots N)$, the decay amplitude into a hadron channel $h\left(e . g ., D^{-} \pi^{+}\right.$or an isospin eigenstate of $\bar{D} \pi$ ) can be expressed as

$$
A(B \rightarrow h)=\sum_{a=1,2, \cdots N} A(B \rightarrow a) e^{i \delta_{a}} O_{h a}
$$

where $O_{h a}$ is the diagonalization matrix between the hadron basis and the eigenchannels:

$$
|h\rangle=\sum_{a} O_{h a}|a\rangle
$$

If the decay occurs through the interactions carrying a common CP-phase (e.g., $\left(\bar{d}_{L} \gamma^{\mu} u_{L}\right)\left(\bar{c}_{L} \gamma_{\mu} b_{L}\right)$ and the interactions arising from the QCD corrections to it), the CPphase factors out: $A(B \rightarrow a)^{*}=A(B \rightarrow a) e^{-2 i \delta_{C P}}$. Unfortunately we have no practical way to solve the multichannel problem for $\delta_{a}$ and $O_{h a}$. If, for instance, the $B^{0} \rightarrow \bar{D} \pi$ amplitude of $I=1 / 2$ involves $N(\gg 1)$ eigenchannels, the $B^{0} \rightarrow D^{-} \pi^{+}$amplitude would also contain roughly as many eigenchannel amplitudes, only by a factor of two or so more. In neither case is the decay phase simply related to the strong-interaction eignephases. That is to say, breaking up the two-body states into the isospin eigenchannels accomplishes very little in relating the decay phases the strong-interaction S-matrix when scattering is highly inelastic. 
Therefore there is no intrinsic merit in studying the phases of the $I=1 / 2$ and $3 / 2$ amplitudes of $B^{0} \rightarrow \bar{D} \pi$ instead of the phases of the $B^{0} \rightarrow D^{-} \pi^{+}$and $B^{0} \rightarrow \bar{D}^{0} \pi^{0}$ amplitudes. It would not be surprising if we have already encountered this situation in the $D$ decay. The $K^{-} \pi^{+}$ channel of the $D^{0}$ decay couples to $\bar{K}^{0} \pi^{0}, \bar{K}^{0} \eta$, and several $\bar{K} \pi \pi \pi$ channels, resonant and nonresonant with different internal quantum numbers. In the past, analysis was made for the isospin amplitudes of $D \rightarrow \bar{K} \pi$ and $K \bar{K}$ []]. In the presence of many other decay channels open, we may equally well present the decay phases for the directly observed amplitudes instead of the isospin amplitudes, particularly in the $B$ decay.

\section{PARAMETRIZATION OF AMPLITUDES AND EXPERIMENTAL DATA}

Since our purpose is to learn about how large the final-state interaction phases are in the $B$ decay, we wish to separate out a CP-phase from the decay amplitudes. For this reason we consider the decay modes in which the nonpenguin interactions dominate. Best measured are the two-body decay modes which are caused by the quark process $b \rightarrow c \bar{u} d$. We analyze four sets of the two-body decay modes:

$$
\left\{\begin{array}{l}
B \rightarrow \bar{D} \pi \\
B \rightarrow \bar{D} \rho \\
B \rightarrow \bar{D}^{*} \pi \\
B \rightarrow \bar{D}^{*} \rho .
\end{array}\right.
$$

Each set consists of three decay modes, for instance, $B^{+} \rightarrow \bar{D}^{0} \pi^{+}, B^{0} \rightarrow D^{-} \pi^{+}$, and $B^{0} \rightarrow \bar{D}^{0} \pi^{0}$ for $B \rightarrow \bar{D} \pi$. All four sets of decays have the same isospin structure. Since the weak hamiltonian transforms like $\Delta I=1$, there are two independent decay amplitudes in each set. Choosing $B \rightarrow \bar{D} \pi$ as an example, we can parametrize the observed amplitudes in terms of the isospin amplitudes as

$$
\begin{aligned}
& A_{0+} \equiv A\left(B^{+} \rightarrow \bar{D}^{0} \pi^{+}\right)=A_{3 / 2} \\
& A_{-+} \equiv A\left(B^{0} \rightarrow D^{-} \pi^{+}\right)=\frac{1}{3}\left(A_{3 / 2}+2 A_{1 / 2}\right) \\
& A_{00} \equiv A\left(B^{0} \rightarrow \bar{D}^{0} \pi^{0}\right)=\frac{\sqrt{2}}{3}\left(A_{3 / 2}-A_{1 / 2}\right)
\end{aligned}
$$

Then the three amplitudes obey the sum rule,

$$
A_{0+}-A_{-+}=\sqrt{2} A_{00}
$$

We denote two relative phases as

$$
\begin{aligned}
\delta_{-+} & =\arg \left(A_{-+} / A_{0+}\right), \\
\delta_{00} & =\arg \left(A_{00} / A_{0+}\right) .
\end{aligned}
$$

With the constraint of the sum rule Eq.(5), the two phases are dependent. We can use alternatively the phase difference of the isospin amplitudes,

$$
\delta_{I}=\arg \left(A_{1 / 2} / A_{3 / 2}\right)
$$


for parametrization.

From the 1998 edition of the Review of Particle Physics [6], we have extracted the magnitudes of amplitude after making the phase space corrections of $p^{2 l+1}$ on the assumption that the $s$-wave dominates in $\bar{D} \pi$ and $\bar{D}^{*} \rho$, and the $p$-wave in $\bar{D}^{*} \pi$ and $\bar{D} \rho$. The results are tabulated in Table I where $\left|A_{-+}\right|$is normalized to unity up to experimental uncertainties. Only upper bounds have been measured for $\left|A_{00}\right|$. We treat the experimental errors for the three amplitudes as uncorrelated. Actually a small portion of the errors $(1 \pm 0.0128)$ in $\left|A_{-+}\right|$and $\left|A_{00}\right|$ comes from a common source, which is the lifetime of $B^{0}$. However, this hardly affects our final numbers.

\section{RESULTS OF ANALYSIS}

The sum rule Eq.(5) can be expressed as a triangular relation in the complex plane for each set of the decay modes. A typical pattern of the triangular relation is depicted in Figure 1, where the phase of $A_{0+}$ is chosen to be zero for reference. $A_{00}$ is confined inside the circle. The sign ambiguity or the phase ambiguity by $\pi$ of $A_{-+} / A_{0+}$ has been fixed such that the three amplitudes be consistent with the sum rule. The sum rule has the ambiguity of the reflection with respect to the real axis. We have fixed this reflection ambiguity or the complex conjugation ambiguity by choosing $\delta_{-+}$between $0^{\circ}$ and $180^{\circ}$. Then $\delta_{00}$ is negative by the sum rule. The bands shown by broken curves at the ends of the arrows indicate the experimental errors. Note that for $A_{-+}$the arrow is attached to the direction of $-_{-+}$. In all cases the triangular relation can be satisfied with zero phases if we take account of the experimental uncertainties. Here we pose the following question: Up to how large phases can be accommodated by the current data if we take the quoted experimental errors seriously?

We have tabulated the answer to the question in Table II. Listed are the bounds on the relative phases $\delta_{-+}$and $\delta_{00}$. In obtaining those bounds, the quoted experimental uncertainties have been taken into account as uncorrelated errors. For comparison, we have also listed the corresponding values for the decay $D \rightarrow \bar{K} \pi$ which has the identical isospin properties as $B \rightarrow \bar{D} \pi$. Its decay interactions are also the same in structure up to the replacement of $b \rightarrow c$ and $c \rightarrow s$. The most important, albeit anticipated, conclusion is that the phase $\delta_{-+}$between $A_{-+}$and $A_{0+}$ must be small in all cases except possibly for $B^{0} \rightarrow \bar{D} \pi$. As the measurement on the branching fractions, particularly of the color-suppressed modes, will improve in the future, either the upper bounds listed in Table II will be tightened or actual values may emerge for $\delta_{-+}$. We are not far from seeing the actual values. In contrast to $\delta_{-+}$, the phase $\delta_{00}$ between $A_{00}$ and $A_{0+}$ is only loosely constrained. The reason is fairly obvious in Figure 1: Though the triangle is very flat, i.e., the final-state interaction is small, the smallness of $\left|A_{00}\right|$ leaves room for the phase of $A_{00}$ to be large. Even if $\delta_{00}$ turns out to be large in the future, it should be interpreted as an accident due to the smallness of $\left|A_{00}\right|$, not as a consequence of large final-state interactions.

We can express the phases in terms of the isospin amplitude phase $\delta_{I}=\delta_{1 / 2}-\delta_{3 / 2}$. In the last column of Table II we have listed $\delta_{I}$. S Since it is $\delta_{-+}$and $\delta_{00}$ that experiment measures

\footnotetext{
${ }^{1}$ In the present phase convention, the bottom entry for $D \rightarrow \bar{K} \pi$ is $\left(97_{-13}^{+12}\right)^{\circ}$ in [0] based on the
} 
directly, the experimental uncertainties accumulate and get enhanced when expressed in $\delta_{I}$.

It is a clear conclusion of our analysis that the final-state interaction is indeed small and the phase must be fairly small at least for $B \rightarrow \bar{D} \pi, \bar{D} \rho$, and $\bar{D}^{*} \rho$. The smallness of the final state interaction phase for the two-body decay was advocated by an intuitive argument based on QCD [2]. It is actually required by the phase-amplitude dispersion relation unless the amplitude is abnormally enhanced or suppressed in magnitude [四]. The possibility that the highly suppressed two-body decay amplitudes such as $A_{00}$ can have large decay phases

has been predicted in the random S-matrix model of the final-state interaction [4]. The smallness of the final-state interaction is a phenomenon special to the two-body decay. It does not imply the same in the multibody or inclusive decays. In the decays where more than two hadrons is produced from a heavy particle, the phase of the decay amplitude depends on the invariant subenergies in the final state. It is almost obvious theoretically that if one or more of the subenergies is small, the phase of the decay amplitude can be large.

To summarize, we have quantified the smallness of the final-state interaction phases which is implied by the color suppression in the $B$ decay. According to the latest worldaverage data, the final-state interaction phases have already been bounded fairly tightly. Our analysis shows that the current bounds on the color-suppressed neutral modes should not be far from their actual values. Lowering the upper bounds on the branching fractions of the color-suppressed modes together with more accurate measurement of the color-favored modes will set even severe limits on the final-state interaction phases or give their actual values. They will have an important implication in the $\mathrm{CP}$ violation search through the modes such as $B \rightarrow \pi \pi$.

\section{ACKNOWLEDGMENTS}

This work is supported in part by the Director, Office of Energy Research, Office of High Energy and Nuclear Physics, Division of High Energy Physics of the U.S. Department of Energy under Contract DE-AC03-76SF00098 and in part by the NSF under grant PHY95-14797.

CLEO data as of 1996 with more generous errors attached. 


\section{REFERENCES}

[1] M. Bander, D. Silvermann, and A. Soni, Phys. Rev. Lett. 43, 242 (1979): J.Gérard and W.-S. Hou, Phys. Rev. Lett. 62855 (1991); Phys. Rev. D 43, 2909 (1991): G. Eilam and D. Wyler, Nucl. Phys. B352, 367 (1991): R. Fleischer, Z. Phys. C 58, 438 (1993); C 6281 (1994): N. Deshpande and X.-G. He, Phys. Lett. B 336, 471 (1994): G. Kramer, W.F. Palmer, and H. Simma, Nucl. Phys. B328, 77 (1994); Z. f. Phys. C 66, 429 (1995).

[2] J.D. Bjorken, Nucl. Phys. B (Proc. Suppl.) 11325 (1989).

[3] H. Zhang, Phys. Lett. B 356, 107 (1995): B. Blok and I. Halperin, Phys. Lett. B 385, 325 (1996): B. Blok, M. Gronau, and J.L. Posner, Phys. Rev. Lett. 78, 3999 (1997): A.N. Kamal and C. W. Luo, Phys. Lett. B 398, 151 (1997): G.Narduli and T.N. Pham, Phys. Lett. B 391, 165 (1997): M. Gronau and J.L. Rosner, Enrico Fermi Institute Preprint, hep-ph/9806348.

[4] M. Suzuki, LBNL Preprint, hep-ph/9807414.

[5] J.F. Donoghue, E. Golowich, A. A. Petrov, and J.M. Soares, Phys. Rev. Lett. 77, 2178 (1996).

[6] Review of Particle Physics, Particle Data Group, C. Caso et al., Eur. Phys. J. C 3, 1 (1998).

[7] CLEO Collaboration, M. Bishai et al., Phys. Rev. Lett. 78, 3261 (1997).

[8] K.M. Watson, Phys. Rev. 95, 228 (1954). See also K. Aizu, Proc. Int, Conf. Theoret. Phys., 1953, Kyoto, Kyoto-Tokyo Science Council, 1954, p.200: E. Fermi, Suppl. Nuovo. Cim. 2, 58 (1955). 


\section{TABLES}

TABLE I. The decay amplitudes extracted from the data. $A_{0+}, A_{-+}$, and $2^{1 / 2} A_{00}$ denote $A\left(B^{+} \rightarrow \bar{D}^{0} \pi^{+}\right), A\left(B^{0} \rightarrow D^{-} \pi^{+}\right)$, and $2^{1 / 2} A\left(B^{0} \rightarrow \bar{D}^{0} \pi^{0}\right)$, respectively, in the case of $B \rightarrow \bar{D} \pi$, and the corresponding amplitudes in other cases. $\left|A_{0+}\right|$ is normalized to unity up to an experimental error.

\begin{tabular}{|c|c|c|c|}
\hline \hline Decay modes & $\left|A_{0+}\right|$ & $\left|A_{-+}\right|$ & $2^{1 / 2}\left|A_{00}\right|$ \\
\hline $\bar{D} \pi$ & $1 \pm 0.0487$ & $0.7741 \pm 0.0526$ & $<0.2188 \pm 0.0028$ \\
\hline $\bar{D} \rho$ & $1 \pm 0.0682$ & $0.7907 \pm 0.0708$ & $<0.2481 \pm 0.0032$ \\
\hline $\bar{D}^{*} \pi$ & $1 \pm 0.0451$ & $0.7976 \pm 0.0320$ & $<0.4497 \pm 0.0058$ \\
\hline $\bar{D}^{*} \rho$ & $1 \pm 0.1007$ & $0.6765 \pm 0.1668$ & $<0.2765 \pm 0.0035$ \\
\hline \hline
\end{tabular}

TABLE II. The bounds on the phases $\delta_{-+}=\arg \left(A_{-+} / A_{0+}\right), \delta_{00}=\arg \left(A_{00} / A_{0+}\right)$, and $\delta_{I}=\arg \left(A_{1 / 2} / A_{3 / 2}\right)$. We have chosen as $0^{\circ}<\delta_{-+}<180^{\circ}$, which leads to $\delta_{00}<0$ and $\delta_{I}>0$.

\begin{tabular}{|c|c|c|c|}
\hline \hline Decay modes & $\delta_{-+}(>0)$ & $\delta_{00}(<0)$ & $\delta_{I}(>0)$ \\
\hline $\bar{D} \pi$ & $<11^{\circ}$ & $>-44^{\circ}$ & $<19^{\circ}$ \\
\hline $\bar{D} \rho$ & $<16^{\circ}$ & $>-60^{\circ}$ & $<26^{\circ}$ \\
\hline $\bar{D}^{*} \pi$ & $<29^{\circ}$ & $>-59^{\circ}$ & $<46^{\circ}$ \\
\hline $\bar{D}^{*} \rho$ & $<21^{\circ}$ & $>-54^{\circ}$ & $<40^{\circ}$ \\
\hline \hline$D \rightarrow \bar{K} \pi$ & $80^{\circ} \pm 7^{\circ}$ & $-70^{\circ} \pm 8^{\circ}$ & $90^{\circ} \pm 7^{\circ}$ \\
\hline \hline
\end{tabular}




\section{FIGURES}

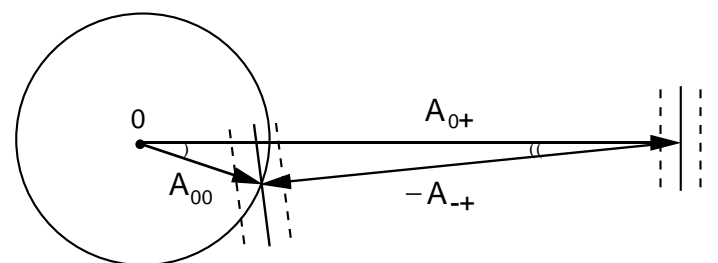

FIG. 1. The sum rule holds in the triangular relation typically as shown here. The phase of $A_{0+}$ has been chosen to be zero for reference. $A_{00}$ is confined inside the circle. The bands indicated by broken lines at the ends of $A_{0+}$ and $-A_{-+}$represent their experimental uncertainties. The upper

bound on $\left|A_{00}\right|$ constrains the angle $\delta_{-+}$between $A_{-+}$and $A_{0+}$ to small values, while the phase of $A_{00}$ is subject to larger uncertainties than that of $A_{-+}$. 